\title{
Random 'spot' urinary metanephrines compared with 24-h-urinary and plasma results in phaeochromocytomas and paragangliomas
}

\author{
Emilia Sbardella1,2, Zoe Maunsell³, Christine J H May1, Michael Tadman'1, Tim James³, \\ Bahram Jafar-Mohammadi ${ }^{1}$, Andrea M Isidori ${ }^{2}$, Ashley B Grossman ${ }^{1}$ and Brian Shine ${ }^{3}$ \\ ${ }^{1}$ Department of Endocrinology, Oxford Centre for Diabetes, Endocrinology and Metabolism, Churchill Hospital, \\ University of Oxford, Oxford, UK, ${ }^{2}$ Department of Experimental Medicine, Sapienza University of Rome, Rome, Italy, \\ and ${ }^{3}$ Department of Clinical Biochemistry, Oxford University Hospitals NHS Foundation Trust, Oxford, UK
}

Correspondence should be addressed to E Sbardella

Email

emi.sbardella@gmail.com

\section{Abstract}

Background: In patients with phaeochromocytomas or paragangliomas (PPGLs), 24-h urine collections for metanephrines (UMNs) are cumbersome.

Objective: To evaluate the diagnostic utility of ratios to creatinine of 'spot' uMNs.

Methods: Concentrations of uMNs and plasma metanephrines (pMNs) were measured by HPLC-mass-spectrometry. We retrospectively compared correlations of 24-h-urine output and ratio to creatinine in historical specimens and prospectively assessed 24-h and contemporaneous spot urines and, where possible, pMNs. Using trimmed logtransformed values, we derived reference intervals based on age and sex for spot urines. We used multiples of upper limit of normal (ULNs) to compare areas under curves (AUCS) for receiver-operator characteristic curves of individual, and sum and product of, components.

Results: In 3143 24-h-urine specimens on 2416 patients, the correlation coefficients between the ratios and outputs of metanephrine, normetanephrine and 3-methoxytyramine in 24-h urines were 0.983, 0.905 and 0.875, respectively. In 96 patients, the correlations between plasma concentrations, urine output and ratios in spot specimens were similar to those for raw output or ratios in 24-h specimens. Of the 160 patients with PPGLs, the Cls for AUCs for individual metabolites overlapped for all four types of measurement, as did those for the sum of the multiple ULNs although these were slightly higher (AUC for spot urine: 0.838 (0.529-1), plasma: 0.929 (0.874-0.984) and output: 0.858 (0.764-0.952)).

Conclusions: Ratios of fractionated metanephrines to creatinine in spot urine samples appear to have a similar diagnostic power to other measurements. The ease of spot urine collection may facilitate diagnosis and follow-up of PPGLs through improved patient compliance.

\section{Introduction}

Phaeochromocytomas and paragangliomas (PPGLs) are rare neuroendocrine tumours that arise from chromaffin cells of the adrenal medulla and extra-adrenal paraganglia, respectively, and may be associated with high morbidity
$(1,2)$. The majority are benign and surgically curable; however, approximately $10 \%$ of phaeochromocytomas and $15-35 \%$ of paragangliomas (PGLs) are invasive or metastatic (1), while up to $40 \%$ of PPGLs are associated 
with an inherited mutation and another $40-50 \%$ of subjects show somatic mutations in one of some 20 susceptibility genes $(3,4,5)$. Therefore, careful long-term follow-up is necessary for patients and, often, for their family members $(3,6,7)$.

Many of these tumours secrete catecholamines (noradrenaline/norepinephrine, adrenaline/epinephrine or dopamine), and these may cause serious and potentially lethal cardiovascular complications, such as hypertensive crises, and may lead to sudden death because of cardiac arrhythmias or by causing stroke. Biochemical testing for a PPGL is indicated not only in symptomatic patients, but also in patients with adrenal incidentalomas or an identified genetic predisposition $(8,9,10,11)$.

Because secretion of catecholamines may be episodic, the concentrations of individual catecholamines may be normal between episodes because of their short halflives. Moreover, they are unstable in plasma and therefore collection and storage of specimens requires exacting conditions. The three main catecholamines (adrenaline, noradrenaline and dopamine) are metabolised to metadrenaline (metanephrine), normetadrenaline (normetanephrine) and 3-methoxytyramine (3MT), respectively. These metabolites are much more stable, and so their concentrations in plasma and excretion in urine are much more constant than the native catecholamines (12). Metanephrines in plasma and urine comprise free and conjugated, mostly sulphated, fractions as reported by Foti and colleagues, the conjugated form predominating in urine. In this study, 'urinary fractionated metanephrines' refers to this mixture (13).

Numerous studies have shown that plasma free or 24-h urinary fractionated metanephrines have high diagnostic sensitivity and specificity and are superior to other tests of catecholamine excess for the diagnosis of phaeochromocytoma $(9,10,14,15,16,17,18,19$, $20,21,22,23,24,25,26,27)$. Specifically, plasma free metanephrines have been reported to show a sensitivity of $97 \%(12,28)$ and a diagnostic specificity between 80 and $100 \%$ (21) in the diagnosis of catecholamine-secreting tumours. However, Perry et al. (29) demonstrated that measurement of 24-h urinary fractionated metanephrines, using mass spectrometry, also shows very high sensitivity (97\%) and specificity (91\%). While various studies have compared the diagnostic accuracy of urinary fractionated metanephrines and plasma free metanephrines using different analytical methodologies, there are no reported comparisons using the gold standard method of liquid chromatography tandem mass spectrometry (LC-MS/ MS) for analysis of urine (30). The concentrations of the metabolites in plasma are much lower than in urine, and therefore, assays for plasma metanephrines are available in fewer centres than urine metanephrines. Moreover, to measure plasma free metanephrines, blood needs to be taken after 30 min of supine rest with an in-dwelling catheter and, ideally, in the fasting state (30), and the specimen needs to be processed within $30 \mathrm{~min}$ and the plasma carefully stored at a low temperature.

Collecting 24-h urine specimens for analysis of urinary fractionated metanephrines is inconvenient and difficult for patients, and the large variation, for instance, in creatinine output in successive 24 -h specimens suggests that many supposed 24-h collections are incomplete or represent collections for more than $24 \mathrm{~h}$. Furthermore, as many patients attend centres of expertise, which may involve considerable travel, 24-h urinary collections may be cumbersome and inconvenient. Since the concentrations of metanephrines in plasma are stable, their secretory rate ought also to be stable. We therefore hypothesised that relating the concentration in urine to creatinine might be suitable for diagnosis, and thus eliminate at least one source of variation. A single 'spot' urine collection corrected for creatinine carried out in the clinic has obvious potential advantages.

In the past, night-time urine collections and 4- to 8-h or single-voided urine collections have been proposed, but these studies were carried out using few patients and older methods for measuring metanephrines (10, 31, 32, 33). More recently, Zuo and colleagues (34) compared free metanephrine concentrations in a spot urine to plasma free metanephrines in 28 patients and 155 control subjects, using HPLC with electrochemical detection, and they reported that the ratio of spot urinary free metanephrines to the urinary creatinine concentration was superior to plasma free metanephrines.

The present study was designed to investigate: first, the correlation between the ratio of metanephrines to creatinine in 24-h specimens and output of metanephrines in 24-urine specimens; second, the correlation between the ratio of metanephrines to creatinine in spot urine specimens, output in 24-h specimens and concentration in plasma samples in patients with and without PPGLs and third, the diagnostic accuracy of spot urinary metanephrines.

\section{Subjects and methods}

The study was in three parts. First, we correlated the results of 24-h output and the ratio between the individual 
components and creatinine, using retrospective data from the laboratory information system. We used data from patients with specimens collected within 30 days of each other to compare the coefficients of variation (CVs) for each component in terms of output and ratio to creatinine.

In the second part, we prospectively asked patients with PPGLs or with clinical conditions that required exclusion of PPGLs (such as adrenal incidentalomas, signs or symptoms typical of PPGLs, genetic mutations associated with PPGLs) attending the Neuroendocrine Tumour Clinic in the Oxford Centre for Diabetes, Endocrinology and Metabolism at the Churchill Hospital, Oxford, UK, to provide us with 24-h urine specimens and then to produce a 'spot' urine $(20 \mathrm{~mL})$ immediately after completing the collection. We also collected plasma specimens for free metanephrines from the majority of these patients. We constructed matrix scatter plots for each metabolite separately and calculated the correlation coefficients using log-transformed values. We also directly compared the ratios of the metabolites to creatinine in spot- and 24-h urine specimens, using a Bland-Altman plot, in which the percentage difference between the two values is plotted against the mean value (35).

In the third part, we retrospectively analysed the diagnostic performance of 'spot' urinary metanephrines, 24-h output and ratios and plasma metanephrines among patients with and without a diagnosis of PPGL, using the first available specimen result. We searched the cellular pathology and radiology databases and the clinical information in the electronic patient records of Oxford University Hospitals for confirmed diagnoses of PPGLs and used only specimens collected before surgery. As controls, we used the earliest specimen from patients who did not have evidence of a diagnosis of PPGL in the clinical details, histology database or radiology database. We used these values to plot receiver-operator characteristic (ROC) curves. We also explored the utility of combinations of the three measurements related to the upper limit of the reference interval, following from our previous work (36).

The first and second parts of the study were conducted using data from September 2015 to December 2018. The third part of the study was conducted using data from January 2017 to December 2018.

In the three study components, we used all available data in our laboratory for specimens collected within 30 days of each other.

In this study, we considered 'spot' urinary metanephrines as the index test, plasma free metanephrines, and urinary fractionated metanephrines as the reference standard according to STARD checklist (see Supplementary STARD checklist, see section on supplementary materials given at the end of this article).

\section{Analytical methods}

Urinary fractionated metanephrines (normetanephrine, metanephrine and 3-methoxytyramine) were analysed by an isotope-dilution HPLC-ECD (Chromsystems Instruments and Methods $\mathrm{GmbH}$, Gräfelfing, Germany) until 2015. The assay was then transferred to an isotopedilution HPLC-tandem mass spectrometric method that was developed and validated in-house. The method is accredited to ISO 15189 by the UK Accreditation Service. This assay incorporates an acid hydrolysis step prior to analysis in order to liberate the analytes from their respective conjugates. Samples were prepared for analysis using solid phase extraction (SPE), dried down under nitrogen then reconstituted in mobile phase. Chromatographic separation was achieved using gradient elution on a Kinetex HILIC HPLC analytical column (2.6 $\mu \mathrm{m} 150 \times 2.1 \mathrm{~mm}$ ) (Phenomenex, Torrance, CA, USA). The coefficients of variation for the three analytes by tandem mass spectrometry were as follows: normetanephrine 5.6\% (95\% CI: 5.05-6.22) at 1.83 and 5.2\% (4.73-5.83\%) at $7.20 \mu \mathrm{mol} / \mathrm{L}$; metanephrine $4.7 \%(4.3-5.3 \%)$ and $4.7 \%$ (4.3-5.3\%) at 0.53 and $2.90 \mu \mathrm{mol} / \mathrm{L}$, respectively and 3MT $10.0 \%(9.1-11.2 \%)$ and $7.0 \%(6.3-7.8 \%)$ at 0.37 and 2.73 $\mu \mathrm{mol} / \mathrm{L}$, respectively. The limit of quantitation for all analytes was $0.05 \mu \mathrm{mol} / \mathrm{L}$ and the limit of detection was $0.02 \mu \mathrm{mol} / \mathrm{L}$.

Creatinine was measured using the Abbott ARCHITECT enzymatic creatinine assay (Abbott Diagnostics). The coefficients of variation for creatinine were 1.5\% (1.4$1.7 \%)$ and $1.4 \%(1.3-1.5 \%)$ at 5.9 and $10.7 \mathrm{mmol} / \mathrm{L}$, respectively, and the limit of quantitation for the assay in urine was $0.19 \mathrm{mmol} / \mathrm{L}$.

Plasma-free metanephrines (normetanephrine, metanephrine and 3-methoxytyramine) were referred to the Royal Victoria Infirmary, Newcastle, UK, where they were analysed by HPLC-tandem mass spectrometry. The coefficients of variation were as follows: normetanephrine $11.0 \% \quad(10.3-11.7 \%), \quad 10.4 \% \quad(9.8-11.1 \%)$ and $7.0 \%$ (6.6-7.5\%) at 620, 2040 and $5103 \mathrm{pmol} / \mathrm{L}$, respectively; metanephrine $\quad 10.0 \% \quad(9.4-10.6 \%), \quad 7.0 \% \quad(6.6-7.4 \%)$ and $7.5 \%(7.0-7.9 \%)$ at 281,1188 and $3839 \mathrm{pmol} / \mathrm{L}$, respectively, and 3MT $14.9 \%$ (14.0-15.9\%), 7.4\% (7.0$7.9 \%)$ and $6.9 \%(6.5-7.4 \%)$ at 168, 1132 and 4512 $\mathrm{pmol} / \mathrm{L}$, respectively. Where concentrations were greater than or less than the reporting limits for the assay, we assigned the reporting limit to the result. Many specimens 
had 3MT concentrations below the reporting level, and this changed part of the way through our study from 180 $\mathrm{pmol} / \mathrm{L}$ to $100 \mathrm{pmol} / \mathrm{L}$.

We recorded the hospital number, sex and date of birth of the patient and the date and time of the specimen, using the finishing time for 24-h specimens or the date and time of receipt if this was not recorded on the specimen bottle. For urine specimens, in addition to the fractionated metanephrines, we measured the creatinine concentration. For timed specimens, we recorded the collection period and volume of the specimen (by weighing - not recorded separately on the laboratory information system).

Patients were not asked to modify their diet prior to specimen collection.

Twenty-four-hour urine specimens and 'spot' urine samples $(20 \mathrm{~mL})$ were collected into plain sterile plastic 24-h urine bottles and universal containers, respectively. Patients in the clinic who participated in this study were asked to collect a 'spot' urine specimen at the end of the 24-h collection. No acid was added to the containers. Blood samples for plasma metanephrines were taken in the supine position with the patient fully recumbent for at least 30 min before sampling.

The criteria used for exclusion of a PPGL were 24-h urinary fractionated metanephrines and/or plasma metanephrines within the reference interval, and no record of histology or radiology results suggesting a diagnosis of PPGL was present. In general, elevated metanephrines more than twice the upper limit of normal were strongly suggestive of a phaeochromocytoma or paraganglioma in an otherwise well patient on no medication and required further investigations such as conventional and nuclear medicine imaging techniques. With regard to imaging techniques, CT is considered the first-choice anatomic imaging modality (or MRI in selected cases); functional imaging such as ${ }^{68} \mathrm{Ga}$-DOTA-TATE, ${ }^{123} \mathrm{I}-\mathrm{MIBG}$ and ${ }^{18} \mathrm{~F}$-DOPA PET scanning can also be used in complex cases.

In cases of borderline-positive results, we optimised sampling conditions, eliminating potential interfering drugs and substances which may be responsible for falsepositive results as previously reported (37).

\section{Ethical approval}

Because the analytical methods were already in use within our laboratory service and all the tests collected were part of clinical care, we did not seek specific Ethics Research Committee approval for this work. However, to ensure it was within an appropriately governed process, it was registered as Service Improvement using the Oxford University Hospitals NHS Foundation Trust Governance Register (register number CSS-BIO-55435).

\section{Statistical analysis}

We used the $\mathrm{R}$ statistical system, version 3.6.3, for data analysis ( $\mathrm{R}$ Core Team 2018. R: A language and environment for statistical computing. $\mathrm{R}$ Foundation for Statistical Computing, Vienna, Austria (URL https:// www.R-project.org/)). In addition to standard libraries, we used the 'tidyverse' library for reading data, data manipulation and producing plots, GGally for producing matrix plots and pROC for receiver-operator characteristic (ROC) analysis.

We calculated the correlations between values for output and ratio of the metabolites to creatinine in 24-h urines, between ratios in spot urine and 24-h urine specimens collected within 60 days of each other and between ratios in spot urine and plasma specimens collected within 60 days of each other. We used the values in spot urines, after logarithmic transformation and trimming of the data using Horn's method (36), to calculate the 95th centile reference intervals for the three metabolites, based on age and sex.

To construct ROC curves, we used the earliest value for each patient and only used the values obtained before surgery for patients with a proven PPG. We converted these values to multiples of the upper limit of the relevant reference interval. We calculated the areas under the curve and 95\% CIs for each parameter. We examined each component separately, the combination of all three values using logistic regression and the sum or product of the multiples of the upper limit of the reference interval (MUL) of all three components or of normetanephrine and metanephrine.

\section{Results}

\section{Part 1: Correlations between the results of 24-h output and the ratio between the individual components and creatinine}

Between January 2015 and December 2018, our laboratory received 3143 24-h urine specimens for metanephrines on 2416 patients (mean age 51.2 years, range 18.0-93.7 years, $57 \%$ females).

The correlation coefficients between the spot ratios and outputs of metanephrine, normetanephrine and 
3-methoxytyramine in 24-h urines were 0.983, 0.905 and 0.875 , respectively (2726 specimens, all $P<0.0001$, Fig. 1 ). The coefficients of variation for specimens collected within 30 days of each other were lower for the ratio measurement for all metabolites than for output values (Table 1). The derived reference intervals are shown in Table 2.

\section{Part 2: Correlations between plasma} concentrations, spot concentrations related to creatinine, urine output and ratio to creatinine in 24-h specimens

We had evaluable data for 96 patients (mean age 53.1 years, range $22.5-88.7$ years, 55\% females), with $91-96$

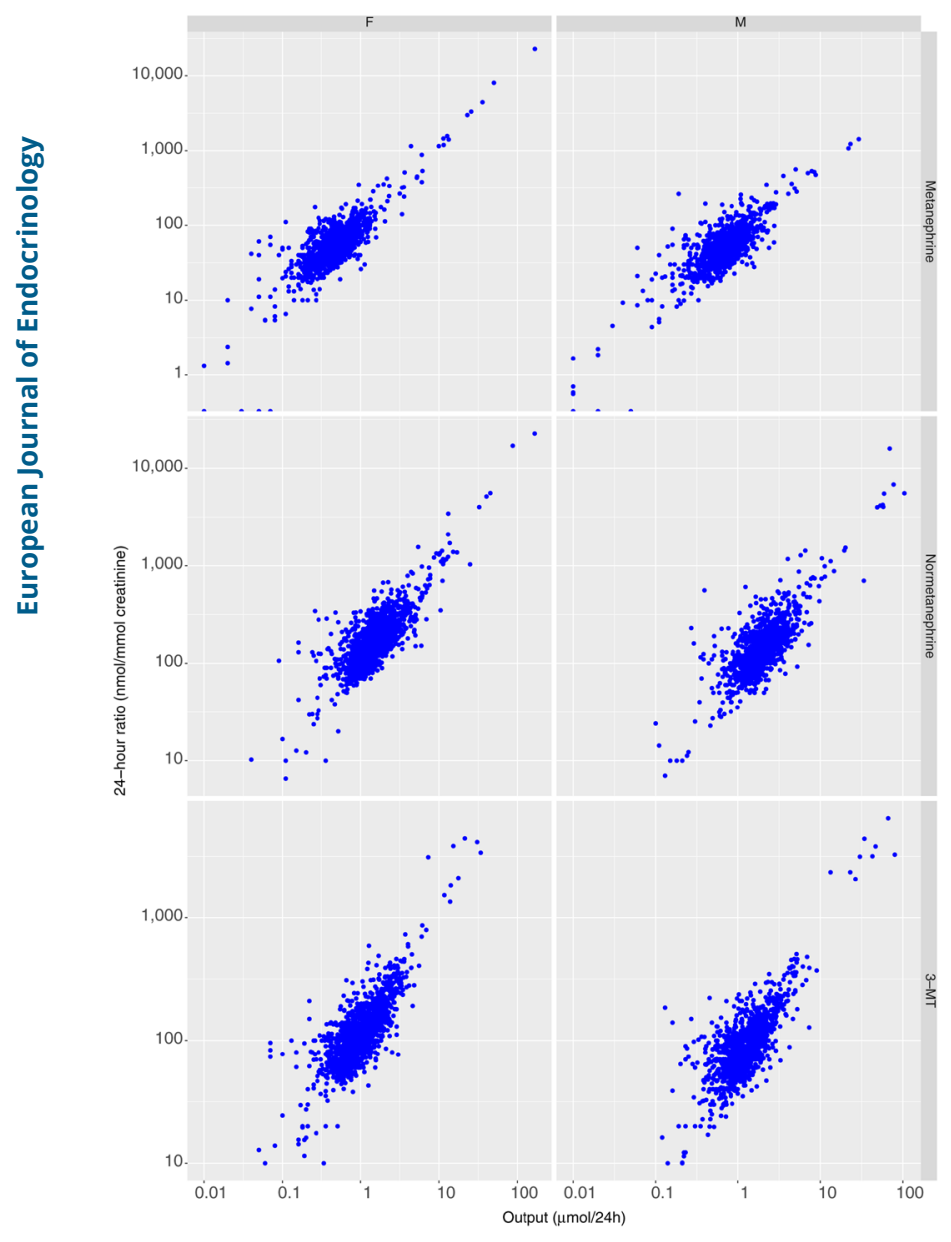

Figure 1

Correlation between raw output values and ratio of concentration to creatinine concentrations of fractionated metanephrines in 24-h urine specimens, subdivided by sex. A full colour version of this figure is available at https://doi. org/10.1530/EJE-19-0809.

Table 1 Coefficients of variation, median (interquartile range), \% of individual metanephrines in 24-h urine for raw output and ratio to creatinine.

Parameter

Metanephrine

Normetanephrine

3-MT

\begin{tabular}{llr}
\hline \multicolumn{1}{c}{ Output } & & \multicolumn{1}{c}{ Ratio } \\
\cline { 1 - 1 } $14.4(6.7-30.5)$ & & $10.5(4.5-22.2)$ \\
$13.4(6-25.4)$ & & $9.4(4.4-17.5)$ \\
$17.7(7.7-33.8)$ & & $13.6(5.9-25.4)$ \\
\hline
\end{tabular}

evaluable pairs of data for each combination of specimen type and metabolite. The median gap between the spot and plasma specimen was 2.9 (IQR 0.4-6.0) days and between spot and 24-h specimen was 2.1 (IQR 1.0-6.0) days. Most spot urines were obtained in the morning, but some were obtained in afternoon clinics (median time of collection $1150 \mathrm{~h}$, IQR 0950-1600 h). The correlations between plasma concentrations, spot concentrations related to creatinine, urine output and ratio to creatinine in 24-h specimens are summarised in Fig. 2A, B and C. Correlations between spot values and plasma values were similar for raw output or ratios in 24-h specimens, but poorer for 3MT. However, many plasma 3MT values were below the reportable minimum (180 pmol/L until July 2017, $100 \mathrm{pmol} / \mathrm{L}$ thereafter). Values for metanephrine and normetanephrine in spot and $24 \mathrm{~h}$ showed excellent correlations (Fig. 2), confirmed by the Bland-Altman plot (Fig. 3).

\section{Part 3: Analysis of the diagnostic performance of 'spot' urinary metanephrines compared with 24-h output and ratios, and plasma metanephrines among patients with and without a diagnosis of PPGLs}

We identified 160 patients with PPGLs (123 patients with phaeochromocytomas, 37 with PGLs, mean age 50.5 years (range 18-83 years), 55\% females). The ROC curves are shown in Fig. 4 and the AUCs, CIs, logistic regression and sum and product of the three measurements in Table 3.

Table 2 Derived reference intervals for ratios of metanephrine components to creatinine $(\mathrm{nmol} / \mathrm{mmol}$ creatinine).

\begin{tabular}{|c|c|c|c|c|c|}
\hline \multirow[b]{2}{*}{ Parameter } & \multirow[b]{2}{*}{ Sex } & \multicolumn{4}{|c|}{ Age (years) } \\
\hline & & $\leq 40$ & $40-60$ & $60-80$ & $>80$ \\
\hline \multirow[t]{2}{*}{ 3MT } & $\mathrm{F}$ & 190 & 220 & 230 & 230 \\
\hline & M & 170 & 200 & 220 & 220 \\
\hline \multirow{2}{*}{ Metanephrine } & $\mathrm{F}$ & 140 & 150 & 170 & 170 \\
\hline & M & 120 & 160 & 170 & 200 \\
\hline \multirow[t]{2}{*}{ Normetanephrine } & $\mathrm{F}$ & 370 & 450 & 500 & 570 \\
\hline & M & 300 & 410 & 450 & 510 \\
\hline
\end{tabular}



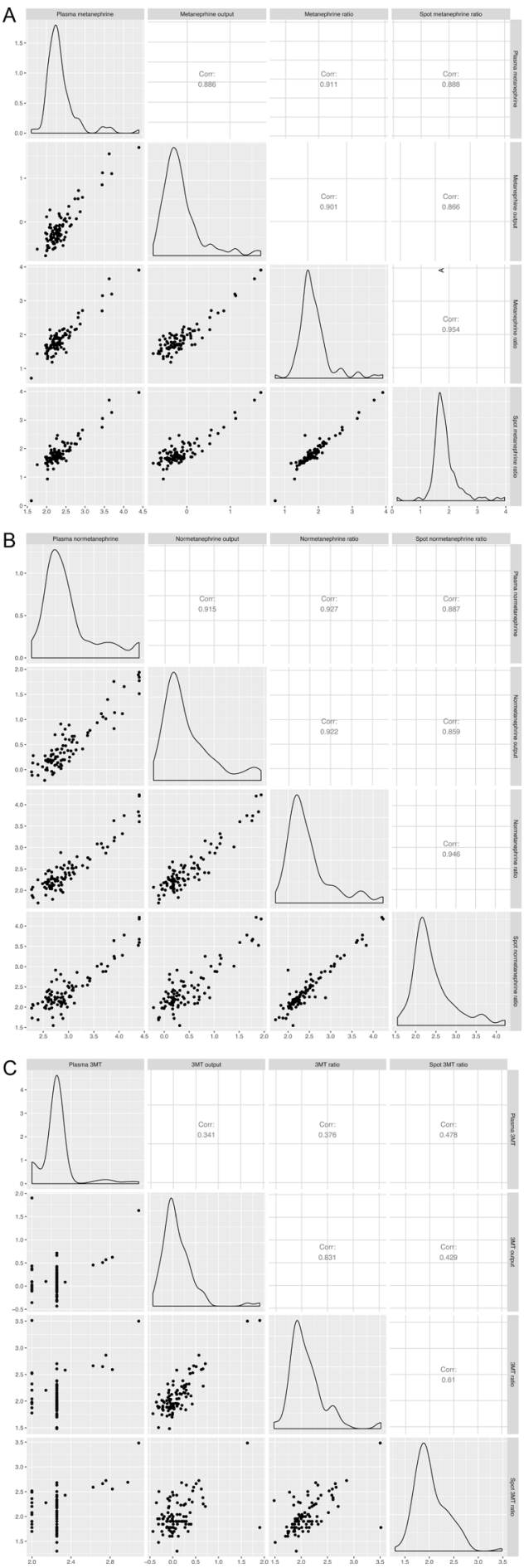

\section{Figure 2}

Scatter plots (below diagonal), correlation (above diagonal) and distribution (diagonal) of log-transformed plasma concentrations, urine outputs in 24-h specimens, ratio to creatinine in 24-h specimens and ratio to creatinine in spot urine specimens for metanephrine $(A)$, normetanephrine $(B)$ and $3 \mathrm{MT}(\mathrm{C})$.

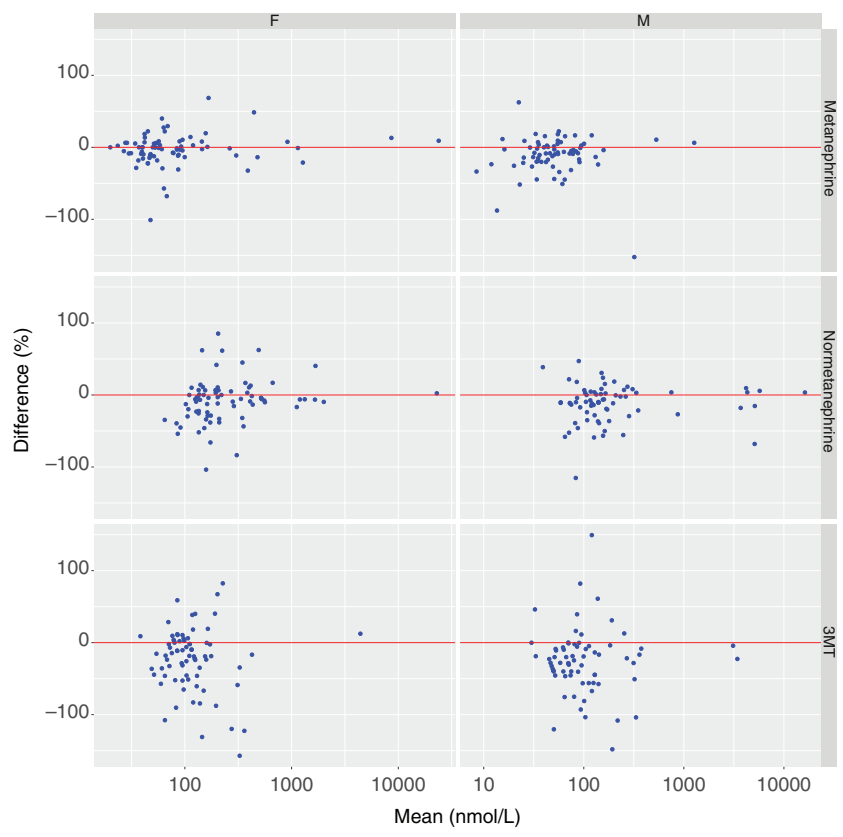

Figure 3

Bland-Altman plot of ratio of fractionated metanephrines to creatinine concentration in spot urine and 24-h urine specimens collected within 30 days of each other. The $X$-axis represents the mean of the two values and the $Y$-axis the percentage difference between the two values. The mean difference and slope were not significant for any of the parameters. A full colour version of this figure is available at https://doi.org/10.1530/EJE-19-0809.

Altogether, 38 patients with PPGLs had valid plasma values, 35 had 24-h output and ratio values and 7 had spot urine ratio values. For each metabolite, the logistic regression and the sums and products of all three components or of normetanephrine and metanephrine, the AUCs were similar for all specimen types, with overlapping CIs.

\section{Discussion}

This is the first study analysing ratios in spot urinary metanephrines and correlations with 24-h urine fractionated metanephrines, assayed by tandem mass spectrometry, and plasma free metanephrines in a large number of patients. We have shown that the ratios of catecholamine metabolites correlate well with the output in 24-h urine specimens (Fig. 1) and that the ratio measurements have lower coefficients of variation than the raw output measurements in repeat specimens collected within 30 days (Table 1). In our prospective study, the ratios in spot urine specimens correlated well 

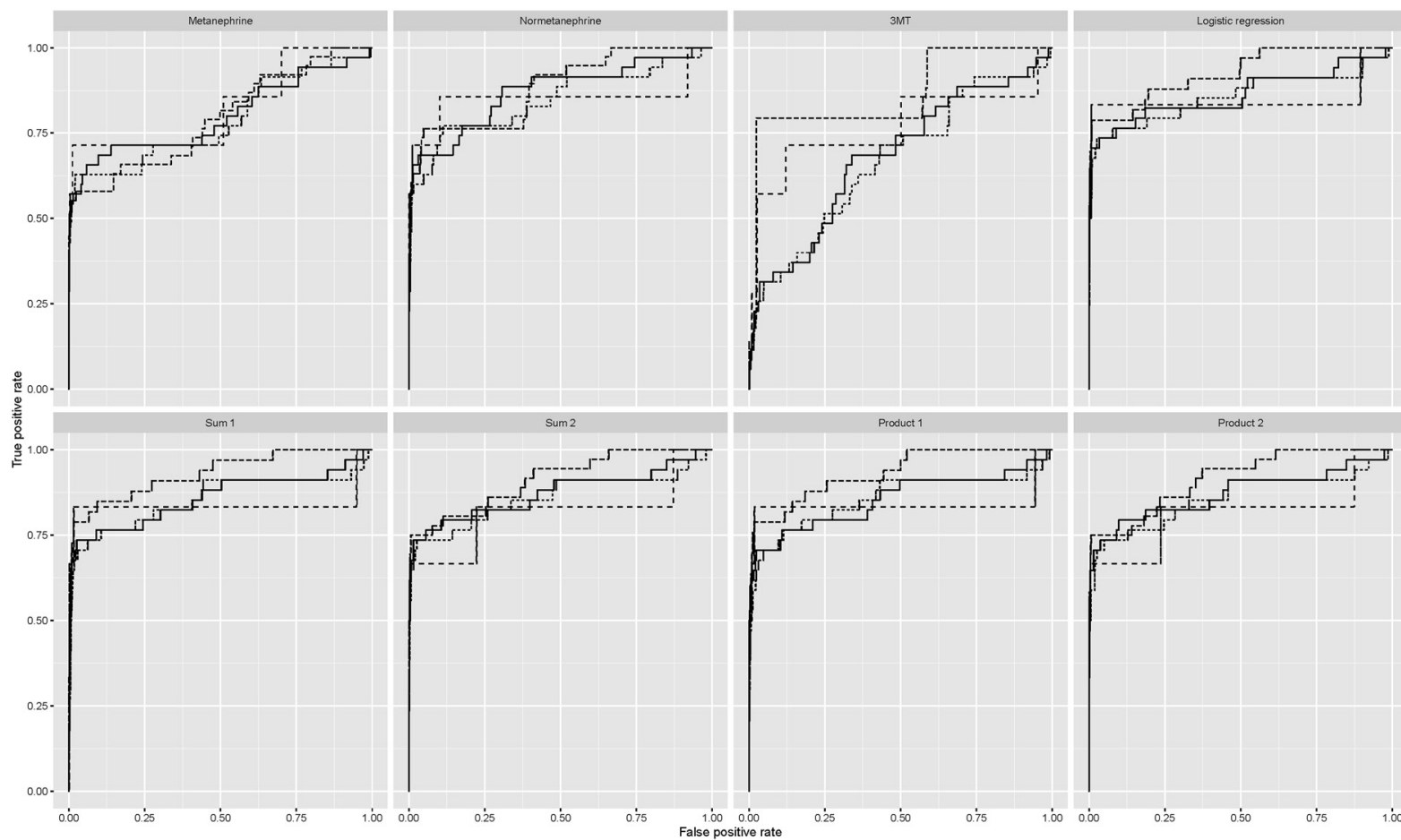

Parameter

- Output

-... Output ratio - - spot

\section{Figure 4}

Receiver operator characteristic (ROC) curves for fractionated metanephrines for plasma concentrations (Plasma), 24-hr output (Output), ratio of concentration to creatinine (Output ratio) in 24-h urine, and ratio of concentration to creatinine in spot urine (Spot), logistic regression for all three measurements, and for sum and product of the three measurements (Sum 1, Product 1) and of metanephrine and normetanephrine (Sum 2, Product 2).

both with the outputs and ratios in 24-h urine specimen and with plasma concentrations, in specimens collected within 60 days of each other (Fig. 2). The values for ratios in spot and 24-h urine specimens also correlated well, and this was confirmed by the Bland-Altman plot (Fig. 3). In the third part of the study, we examined the diagnostic performance of the four types of measurement and found that the areas under the curve were similar, with overlapping CIs for output and ratio in 24-h specimens, spot urine, and plasma specimens for each analyte and similar sensitivity and specificity for a cut-off value at the upper limit of the reference interval (Fig. 4 and Table 3). In addition, we examined the utility of logistic regression and the sums and products of MULs. Plasma values provided the best discrimination for normetanephrine and for all combinations (logistic, sums and products), but with overlapping CIs for all measurements and combinations. 3MT alone had

Table 3 Areas under the curve (AUC, 95\% Cls) for Receiver Operator Characteristic curves for fractionated metanephrines, logistic regression, sum and product of the three measurements (Sum 1, Product 1) and of metanephrine and normetanephrine (Sum 2, Product 2).

\begin{tabular}{lcc}
\hline Parameter & & Output \\
\cline { 1 - 1 } Metanephrine & & $0.798(0.693-0.902)$ \\
Normetanephrine & $0.87(0.792-0.949)$ \\
3MT & & $0.679(0.577-0.78)$ \\
Logistic regression & & $0.863(0.77-0.956)$ \\
Sum 1 & & $0.858(0.764-0.952)$ \\
Sum 2 & $0.873(0.783-0.962)$ \\
Product 1 & $0.853(0.759-0.948)$ \\
Product 2 & $0.871(0.782-0.961)$ \\
\hline
\end{tabular}

\begin{tabular}{l}
\hline \multicolumn{1}{c}{ Output ratio } \\
\hline $0.784(0.681-0.888)$ \\
$0.846(0.756-0.935)$ \\
$0.667(0.563-0.77)$ \\
$0.857(0.761-0.954)$ \\
$0.853(0.756-0.95)$ \\
$0.859(0.764-0.954)$ \\
$0.855(0.759-0.95)$ \\
$0.857(0.763-0.951)$ \\
\hline
\end{tabular}

\begin{tabular}{llll}
\cline { 1 - 1 } \multicolumn{1}{c}{ Plasma } & & \multicolumn{1}{c}{ Spot } \\
\hline $0.791(0.7-0.882)$ & & $0.814(0.579-1)$ \\
$0.883(0.817-0.948)$ & & $0.851(0.594-1)$ \\
$0.679(0.599-0.758)$ & & $0.759(0.485-1)$ \\
$0.925(0.87-0.98)$ & & $0.848(0.557-1)$ \\
$0.929(0.874-0.984)$ & & $0.838(0.529-1)$ \\
$0.912(0.853-0.97)$ & & $0.816(0.537-1)$ \\
$0.931(0.88-0.982)$ & & $0.838(0.531-1)$ \\
$0.915(0.861-0.969)$ & & $0.813(0.531-1)$ \\
\hline
\end{tabular}


the lowest discriminatory value for all measurement methods (Fig. 4 and Table 3).

As controls, we used data of patients with 24-h urinary fractionated metanephrines and/or plasma metanephrines within the reference interval and with no histological or radiology records suggesting a diagnosis of PPGL. Many of these patients were undergoing testing because of hypertension, but some had adrenal incidentalomas, signs or symptoms suggestive of PPGLs or genetic mutations associated with PPGLs.

The Endocrine Society Clinical Practice Guidelines on PPGLs (30) recommend inclusion of measurement of plasma free or urine fractionated metanephrines to test for PPGLs producing catecholamines. Measurements of urinary fractionated metanephrines have a broadly similar diagnostic sensitivity to plasma free metanephrines, although the diagnostic specificity is slightly lower with a higher false positive rate $(30,38)$. In their review, Lenders and colleagues (30) report that numerous studies have confirmed the higher diagnostic sensitivity and advantages of plasma free metanephrines over other tests for detection of PPGLs. Several of these studies involved comparisons of plasma free with urinary fractionated metanephrines (21). Although all yielded consistently higher values for diagnostic sensitivity with the plasma than with the urine test, the differences were small and did not reach statistical significance; moreover, all these studies had limitations and none involved head-to-head comparisons of mass spectrometry-based measurements that represent the gold standard technique. It thus remains a matter of debate whether one test is superior to the other (39), and the choice may depend on the availability at a given site.

While the plasma test may offer advantages over the urine test, these are offset by the need for patient preparation (fasting, supine for 30 min before sampling) and for the specimen to be spun and frozen within 30 $\min (30,36)$. For this reason, urine tests have remained the mainstay of clinical practice in the United Kingdom. Moreover, because the concentrations of the metabolites are lower in plasma, most countries have more laboratories that can measure urinary-fractionated metanephrines than that can analyse plasma free metanephrines.

Collecting 24-h urine specimens has several disadvantages: it is challenging and inconvenient; specimens need to be stored before being sent to the laboratory; the timing of many collections is inaccurate, as judged by creatinine output and the patient may have to return to the clinic or laboratory with the specimen. While shorter periods of sample collection, such as night- time urine collections, 4- to 8-h or single voided 1-h urine collections have been proposed in the past $(10,26,31,32$, $33,40)$, they have not found general favour.

By contrast, spot urines are easy to obtain and transport to the laboratory and do not require special processing, since all the metabolites and creatinine are stable in the time frame for reaching the laboratory. Because the specimen can be collected when the patient is in the clinic, the results may be available sooner. Zuo and colleagues (34) measured free metanephrines concentrations in spot urine and plasma of PPGLs patients and control subjects by HPLC with electrochemical detection and showed that the ratio of spot urinary free metanephrines-to-creatinine ratios were superior to plasma free metanephrines, but they did not consider 3-methoxytyramine (although this is often of less diagnostic significance for adults (41)) and they did not compare these specimens with 24-h urine specimens. Moreover, they analysed spot urinary free metanephrines rather than spot urinary fractionated total metanephrines as we did.

The interest in simplifying the collection of urinary metanephrines using sample spots is also demonstrated by very recent work by Takekoshi and colleagues (42), where creatinine-corrected metanephrine fractions in spot urine and 24-h urine samples from 16 patients were compared to assess the therapeutic effect of metyrosine. They showed that correlations and ROCs between 24-h urinary vanillylmandelic acid, adrenaline and noradrenaline and 24-h urinary metanephrine or urinary normetanephrine were similar to those between spot urinary metanephrine or urinary normetanephrine and 24-h urinary metanephrine or urinary normetanephrine. Moreover, they observed no significant differences between spot and 24-h urine for the assessment of metyrosine treatment by quantifying urinary metanephrine or urinary normetanephrine in Japanese patients with PPGLs, suggesting that spot urine samples could represent a useful tool to evaluate the therapeutic effect of metyrosine in patients with PPGLs.

Besides diagnosis, spot urine specimens may make follow-up of PPGLs easier, since this group of patients should be screened annually for at least 10 years following initial surgery for local or metastatic recurrences or new tumours (43). Annual follow-up for life is required for high-risk patients (young patients and those with a genetic disease, a large tumour and/or a paraganglioma) (44).

Finally, clinicians are interested in rules that may help them to make or exclude a diagnosis. We have previously (37) suggested that the product of MULs for output values has high discrimination. This idea has also been 
explored by Rao (45), who reported a sensitivity of $98.6 \%$ and specificity of $95.1 \%$ for PPGLs, with a raised value in plasma for any of the three components. In this study, the sum and products of MULs had higher discriminatory ability than the individual components, although all CIs overlapped.

\section{Limitations and further perspectives}

The principal limitation of this study is our own institutional referral bias that may have lead to a potential positive selection bias that may have over-estimated the prevalence rate of more aggressive PPGLs. Another limitation is represented by the relatively small number of patients who had PPGLs.

Further perspective studies in a larger group of patients with PPGLs would be needed to confirm these results.

\section{Conclusions}

Ratios of fractionated metanephrines to creatinine in spot urine samples appear to have a similar diagnostic power to the other three measurements, and the ease of spot urine collection may facilitate the diagnosis and follow up of PPGLs through improved patient compliance. While this study was not designed to demonstrate equivalence of the various techniques, it does suggest that there is a clinical utility in the assessment of patients with phaeochromocytomas or paragangliomas, where appropriate.

We have shown that spot urine specimens may have the potential to offer a viable alternative to both 24 -h urine collection and plasma for assessment of production of the catecholamine metabolites, normetanephrine, metanephrine and 3-methoxytyramine. The ratios of normetanephrine, metanephrine and 3-methoxytyramine to creatinine in spot urine samples correlate well with the output and ratios to creatinine of these metabolites in a 24-h collection, although less well with plasma concentrations. Spot urine specimens may, in the future, simplify diagnosis and follow-up of PPGLs, thereby increasing patient compliance in certain situations.

Supplementary materials

This is linked to the online version of the paper at https://doi.org/10.1530/ EJE-19-0809.

\section{Declaration of interest}

Andrea Isidori is on the editorial board of European Journal of Endocrinology. Andrea Isidori was not involved in the review or editorial process for this paper, on which she is listed as an author. The other authors have nothing to disclose.

\section{Funding}

No funding was used to conduct this study and to produce this manuscript.

\section{Data availability}

The datasets generated during and/or analysed during the current study are not publicly available but are available from the corresponding author on reasonable request.

\section{References}

1 Chrisoulidou A, Kaltsas G, Ilias I \& Grossman AB. The diagnosis and management of malignant phaeochromocytoma and paraganglioma. Endocrine-Related Cancer 200714 569-585. (https://doi.org/10.1677/ ERC-07-0074)

2 Nolting S, Grossman A \& Pacak K. Metastatic phaeochromocytoma: spinning towards more promising treatment options. Experimental and Clinical Endocrinology and Diabetes 2019127 117-128. (https:// doi.org/10.1055/a-0715-1888)

3 Sbardella E, Cranston T, Isidori AM, Shine B, Pal A, JafarMohammadi B, Sadler G, Mihai R \& Grossman AB. Routine genetic screening with a multi-gene panel in patients with pheochromocytomas. Endocrine 201859 175-182. (https://doi. org/10.1007/s12020-017-1310-9)

4 Burnichon N, Vescovo L, Amar L, Libe R, de Reynies A, Venisse A, Jouanno E, Laurendeau I, Parfait B, Bertherat J et al. Integrative genomic analysis reveals somatic mutations in pheochromocytoma and paraganglioma. Human Molecular Genetics 201120 3974-3985. (https://doi.org/10.1093/hmg/ddr324)

5 Fishbein L, Leshchiner I, Walter V, Danilova L, Robertson AG, Johnson AR, Lichtenberg TM, Murray BA, Ghayee HK, Else T et al. Comprehensive molecular characterization of pheochromocytoma and paraganglioma. Cancer Cell 201731 181-193. (https://doi. org/10.1016/j.ccell.2017.01.001)

6 Andrews KA, Ascher DB, Pires DEV, Barnes DR, Vialard L, Casey RT, Bradshaw N, Adlard J, Aylwin S, Brennan P et al. Tumour risks and genotype-phenotype correlations associated with germline variants in succinate dehydrogenase subunit genes SDHB, SDHC and SDHD. Journal of Medical Genetics 201855 384-394. (https://doi. org/10.1136/jmedgenet-2017-105127)

7 Gunawardane PTK \& Grossman A. Phaeochromocytoma and paraganglioma. Advances in Experimental Medicine and Biology 2017 956 239-259. (https://doi.org/10.1007/5584_2016_76)

8 Lenders JW, Eisenhofer G, Mannelli M \& Pacak K. Phaeochromocytoma. Lancet 2005366 665-675. (https://doi. org/10.1016/S0140-6736(05)67139-5)

9 Plouin PF, Duclos JM, Menard J, Comoy E, Bohuon C $\&$ Alexandre JM. Biochemical tests for diagnosis of phaeochromocytoma: urinary versus plasma determinations. BMJ 1981282 853-854. (https://doi.org/10.1136/bmj.282.6267.853)

10 Gitlow SE, Mendlowitz M \& Bertani LM. The biochemical techniques for detecting and establishing the presence of a pheochromocytoma. A review of ten years' experience. American Journal of Cardiology 1970 26 270-279. (https://doi.org/10.1016/0002-9149(70)90794-0)

11 Sbardella E, Minnetti M, D’Aluisio D, Rizza L, Di Giorgio MR, Vinci F, Pofi R, Giannetta E, Venneri MA, Vestri A et al. 
Cardiovascular features of possible autonomous cortisol secretion in patients with adrenal incidentalomas. European Journal of Endocrinology 2018178 501-511. (https://doi.org/10.1530/EJE-170986)

12 Eisenhofer G, Prejbisz A, Peitzsch M, Pamporaki C, Masjkur J, Rogowski-Lehmann N, Langton K, Tsourdi E, Pęczkowska M, Fliedner $\mathrm{S}$ et al. Biochemical diagnosis of chromaffin cell tumors in patients at high and low risk of disease: plasma versus urinary free or deconjugated O-methylated catecholamine metabolites. Clinical Chemistry 201864 1646-1656. (https://doi.org/10.1373/ clinchem.2018.291369)

13 Foti A, Adachi M \& DeQuattro V. The relationships of free to conjugated normetanephrine in plasma and spinal fluid of hypertensive patients. Journal of Clinical Endocrinology and Metabolism 198255 81-85. (https://doi.org/10.1210/jcem-55-1-81)

14 Eisenhofer G, Pacak K, Huynh TT, Qin N, Bratslavsky G, Linehan WM, Mannelli M, Friberg P, Grebe SK, Timmers HJ et al. Catecholamine metabolomic and secretory phenotypes in phaeochromocytoma. Endocrine-Related Cancer 201118 97-111. (https://doi.org/10.1677/ERC-10-0211)

15 Lenders JW, Eisenhofer G, Armando I, Keiser HR, Goldstein DS $\&$ Kopin IJ. Determination of metanephrines in plasma by liquid chromatography with electrochemical detection. Clinical Chemistry 199339 97-103. (https://doi.org/10.1093/clinchem/39.1.97)

16 Eisenhofer G, Huynh TT, Hiroi M \& Pacak K. Understanding catecholamine metabolism as a guide to the biochemical diagnosis of pheochromocytoma. Reviews in Endocrine and Metabolic Disorders 20012 297-311. (https://doi.org/10.1023/a:1011572617314)

17 Gardet V, Gatta B, Simonnet G, Tabarin A, Chene G, Ducassou D $\&$ Corcuff JB. Lessons from an unpleasant surprise: a biochemical strategy for the diagnosis of pheochromocytoma. Journal of Hypertension 200119 1029-1035. (https://doi.org/10.1097/00004872200106000-00006)

18 Gerlo EA \& Sevens C. Urinary and plasma catecholamines and urinary catecholamine metabolites in pheochromocytoma: diagnostic value in 19 cases. Clinical Chemistry 199440 250-256. (https://doi.org/10.1093/clinchem/40.2.250)

19 Grossman A, Pacak K, Sawka A, Lenders JW, Harlander D, Peaston RT, Reznek R, Sisson J \& Eisenhofer G. Biochemical diagnosis and localization of pheochromocytoma: can we reach a consensus? Annals of the New York Academy of Sciences 20061073 332-347. (https://doi.org/10.1196/annals.1353.038)

20 Sawka AM, Prebtani AP, Thabane L, Gafni A, Levine M \& Young Jr WF. A systematic review of the literature examining the diagnostic efficacy of measurement of fractionated plasma free metanephrines in the biochemical diagnosis of pheochromocytoma. BMC Endocrine Disorders 20044 2. (https://doi.org/10.1186/1472-6823-4-2)

21 Lenders JW, Pacak K, Walther MM, Linehan WM, Mannelli M, Friberg P, Keiser HR, Goldstein DS \& Eisenhofer G. Biochemical diagnosis of pheochromocytoma: which test is best? JAMA $2002 \mathbf{2 8 7}$ 1427-1434. (https://doi.org/10.1001/jama.287.11.1427)

22 Sawka AM, Jaeschke R, Singh RJ \& Young Jr WF. A comparison of biochemical tests for pheochromocytoma: measurement of fractionated plasma metanephrines compared with the combination of 24-hour urinary metanephrines and catecholamines. Journal of Clinical Endocrinology and Metabolism 200388 553-558. (https://doi. org/10.1210/jc.2002-021251)

23 Davidson DF. Phaeochromocytoma with normal urinary catecholamines: the potential value of urinary free metadrenalines. Annals of Clinical Biochemistry 200239 557-566. (https://doi. org/10.1177/000456320203900603)

24 Plouin PF, Amar L, Dekkers OM, Fassnacht M, Gimenez-Roqueplo AP, Lenders JW, Lussey-Lepoutre C, Steichen O \& Guideline Working Group. European Society of Endocrinology Clinical Practice Guideline for long-term follow-up of patients operated on for a phaeochromocytoma or a paraganglioma. European Journal of
Endocrinology 2016174 G1-G10. (https://doi.org/10.1530/EJE-160033)

25 Sheps SG, Tyce GM, Flock EV \& Maher FT. Current experience in the diagnosis of pheochromocytoma. Circulation 196634 473-483. (https://doi.org/10.1161/01.cir.34.3.473)

26 Bravo EL \& Gifford Jr RW. Current concepts. Pheochromocytoma: diagnosis, localization and management. New England Journal of Medicine 1984311 1298-1303. (https://doi.org/10.1056/ NEJM198411153112007)

27 Grouzmann E, Drouard-Troalen L, Baudin E, Plouin PF, Muller B, Grand D \& Buclin T. Diagnostic accuracy of free and total metanephrines in plasma and fractionated metanephrines in urine of patients with pheochromocytoma. European Journal of Endocrinology 2010162 951-960. (https://doi.org/10.1530/EJE-09-0996)

28 Lenders JW, Keiser HR, Goldstein DS, Willemsen JJ, Friberg P, Jacobs MC, Kloppenborg PW, Thien T \& Eisenhofer G. Plasma metanephrines in the diagnosis of pheochromocytoma. Annals of Internal Medicine 1995123 101-109. (https://doi.org/10.7326/00034819-123-2-199507150-00004)

29 Perry CG, Sawka AM, Singh R, Thabane L, Bajnarek J \& Young Jr WF. The diagnostic efficacy of urinary fractionated metanephrines measured by tandem mass spectrometry in detection of pheochromocytoma. Clinical Endocrinology 200766 703-708. (https://doi.org/10.1111/j.1365-2265.2007.02805.x)

30 Lenders JW, Duh QY, Eisenhofer G, Gimenez-Roqueplo AP, Grebe SK, Murad MH, Naruse M, Pacak K, Young WF \& Endocrine Society. Pheochromocytoma and paraganglioma: an Endocrine Society clinical practice guideline. Journal of Clinical Endocrinology and Metabolism 201499 1915-1942. (https://doi.org/10.1210/jc.20141498)

31 Sullivan JM \& Solomon HS. The diagnosis of pheochromocytoma. Overnight excretion of catecholamine metabolites. JAMA 1975231 618-619. (https://doi.org/10.1001/jama.1975.03240180054017)

32 Ganguly A, Henry DP, Yune HY, Pratt JH, Grim CE, Donohue JP \& Weinberger MH. Diagnosis and localization of pheochromocytoma. Detection by measurement of urinary norepinephrine excretion during sleep, plasma norepinephrine concentration and computerized axial tomography (CT-scan). American Journal of Medicine 197967 21-26. (https://doi.org/10.1016/00029343(79)90064-0)

33 Kaplan NM, Kramer NJ, Holland OB, Sheps SG \& Gomez-Sanchez C. Single-voided urine metanephrine assays in screening for pheochromocytoma. Archives of Internal Medicine 1977137 190-193. (https://doi.org/10.1001/archinte.1977.03630140040011)

34 Zuo M, Zhen Q, Zhang X, Zou W, Yang X, Tian G, Shi Z, Li Q \& Ding M. High specificity of spot urinary free metanephrines in diagnosis and prognosis of pheochromocytomas and paragangliomas by HPLC with electrochemical detection. Clinica Chimica Acta: International Journal of Clinical Chemistry 2018478 82-89. (https:// doi.org/10.1016/j.cca.2017.12.026)

35 Bland JM \& Altman DG. Statistical methods for assessing agreement between two methods of clinical measurement. Lancet 19861 307-310. (https://doi.org/10.1016/j.ijnurstu.2009.10.001)

36 Horn PS, Feng L, Li Y \& Pesce AJ. Effect of outliers and nonhealthy individuals on reference interval estimation. Clinical Chemistry 2001 47 2137-2145. (https://doi.org/10.1093/clinchem/47.12.2137)

37 Sbardella E \& Grossman AB. Pheochromocytoma: an approach to diagnosis. Best Practice and Research: Clinical Endocrinology and Metabolism 202034 101346. (https://doi.org/10.1016/j. beem.2019.101346)

38 Brain KL, Kay J \& Shine B. Measurement of urinary metanephrines to screen for pheochromocytoma in an unselected hospital referral population. Clinical Chemistry 200652 2060-2064. (https://doi. org/10.1373/clinchem.2006.070805)

39 Hickman PE, Leong M, Chang J, Wilson SR \& McWhinney B. Plasma free metanephrines are superior to urine and plasma catecholamines 
and urine catecholamine metabolites for the investigation of phaeochromocytoma. Pathology 200941 173-177. (https://doi. org/10.1080/00313020802579284)

40 Oishi S, Sasaki M, Ohno M \& Sato T. Urinary normetanephrine and metanephrine measured by radioimmunoassay for the diagnosis of pheochromocytoma: utility of 24-hour and random 1-hour urine determinations. Journal of Clinical Endocrinology and Metabolism 1988 67 614-618. (https://doi.org/10.1210/jcem-67-3-614)

41 Eisenhofer G, Goldstein DS, Walther MM, Friberg P, Lenders JW, Keiser HR \& Pacak K. Biochemical diagnosis of pheochromocytoma: how to distinguish true- from false-positive test results. Journal of Clinical Endocrinology and Metabolism 200388 2656-2666. (https:// doi.org/10.1210/jc.2002-030005)

42 Takekoshi K, Satoh F, Tanabe A, Okamoto T, Ichihara A, Tsuiki M, Katabami T, Nomura M, Tanaka T, Matsuda T et al. Correlation between urinary fractionated metanephrines in 24-hour and spot urine samples for evaluating the therapeutic effect of Metyrosine: a subanalysis of a multicenter, open-label phase I/II study. Endocrine
Journal 201966 1063-1072. (https://doi.org/10.1507/endocrj.EJ190125)

43 Balla A, Ortenzi M, Palmieri L, Corallino D, Meoli F, Ursi P, Puliani G, Sbardella E, Isidori AM, Guerrieri M et al. Laparoscopic bilateral anterior transperitoneal adrenalectomy: 24 years experience. Surgical Endoscopy 201933 3718-3724. (https://doi.org/10.1007/s00464-01906665-6)

44 NöltingS, Ullrich M, Pietzsch J, Ziegler CG, Eisenhofer GA \& Pacak K. Current management of pheochromocytoma/ paraganglioma: a guide for the practicing clinician in the era of precision medicine. Cancers 201911 1505. (https://doi.org/10.3390/ cancers11101505)

45 Rao D, Peitzsch M, Prejbisz A, Hanus K, Fassnacht M, Beuschlein F, Brugger C, Fliedner S, Langton K, Pamporaki C et al. Plasma methoxytyramine: clinical utility with metanephrines for diagnosis of pheochromocytoma and paraganglioma. European Journal of Endocrinology 2017177 103-113. (https://doi.org/10.1530/EJE-170077)

Received 10 October 2019

Revised version received 4 May 2020

Accepted 15 May 2020 